\title{
Stenting with high-intensity focused ultrasound ablation for distal biliary obstruction caused by pancreatic carcinoma: a meta-analysis
}

\author{
Xin-Qiang Liư ${ }^{1}$, Sha-Sha Cui ${ }^{2}$, Yu-Ling Kan², Jia-Fen Wang ${ }^{2}$, Yuan-Shun Xü ${ }^{3}$, Feng-Qin Zhang ${ }^{4}$, Pan-Hao Rong ${ }^{3}$ \\ ${ }^{1}$ Department of Oncology, Binzhou People's Hospital, Binzhou, China \\ 2Department of Clinical Laboratory, Binzhou People's Hospital, Binzhou, China \\ ${ }^{3}$ Department of Radiology, Xuzhou Central Hospital, Xuzhou, China \\ ${ }^{4}$ Department of Ultrasound, Binzhou Medical University Hospital, Binzhou, China
}

Gastroenterology Rev 2022; 17 (2): 116-122

DOI: https://doi.org/10.5114/pg.2021.107798

Key words: stent, high-intensity focused ultrasound, pancreatic carcinoma.

Address for correspondence: Yuan-Shun Xu, Department of Radiology, Xuzhou Central Hospital, Xuzhou, China,

e-mail: xuyuanshun1980@163.com

\begin{abstract}
Introduction: Between $42 \%$ and $77 \%$ of patients with distal malignant biliary obstruction (MBO) suffer from pancreatic carcinoma $(P C)$.

Aim: To analyse the clinical efficacy of stenting accompanied by high-intensity focused ultrasound (HIFU) ablation in patients with distal MBO from PC.

Material and methods: Relevant articles published through March 2021 were identified in the Pubmed, Cochrane Library, Embase, Wanfang, VIP, and CNKI databases. RevMan v5.3 and Stata v12.0 were used for the meta-analysis.

Results: Twenty-nine articles were initially identified, and 5 of these were eventually included. These articles described 142 patients who underwent biliary stenting alone and 132 patients who underwent biliary stenting with HIFU ablation. The pooled $\Delta$ total bilirubin (TBIL) values were comparable between the 2 treatment groups $(p=0.10)$. The pooled stent dysfunction rate was significantly greater in the group with stenting alone $(p=0.03)$, and the pooled HR for the stent patency duration indicated that the duration of stent patency was increased in the stenting with HIFU ablation group $(p<0.0001)$. Overall survival rates were significantly longer in the stenting with HIFU ablation group $(p<0.0001)$. HIFU ablation was associated with an $80 \%$ pooled clinical response rate. The pooled cholangitis $(p=0.47)$ and pancreatitis $(p=0.56)$ rates were comparable between the 2 groups. Funnel plots did not reveal any significant evidence of endpoint-associated publication bias.

Conclusions: Stenting with HIFU ablation increased both stent patency and overall survival in patients with distal MBO caused by PC compared to stenting alone.
\end{abstract}

\section{Introduction}

Distal malignant biliary obstruction (MBO) is usually caused by primary biliary and pancreatic tumours [1-5]. Between $42 \%$ and $77 \%$ of patients with distal MBO suffer from pancreatic carcinoma (PC) $[4,6,7]$. PC itself is a highly malignant tumour; moreover, when it is the cause of distal MBO, surgical resection is only possible in a minority (10-20\%) of cases, and even then the 3- and 5-year survival rates of these patients are just $18-52 \%$ and $5-31 \%$, respectively [8]. When patients are diagnosed with MBO caused by PC, only palliative treatment options are feasible.
At present, endoscopic or percutaneous metal stenting is the first choice for patients with distal MBO caused by PC $[9,10]$. However, stenting alone cannot treat or cure the $\mathrm{PC}$, and tumour progression usually limits both the stent patency and the long-term overall survival (OS) of the patient. Therefore, anticancer treatments in addition to stenting should be used for patients with distal MBO caused by PC. Several anticancer treatments, including chemotherapy, radiotherapy, and combination chemoradiotherapy, are used to extend both stent patency and patient OS [8-10]. At present, advances in the use of high-intensity focused ultrasound (HIFU) ablation in addition to stenting to 
treat PC have been made owing to the non-invasive and non-toxic nature of this therapeutic modality [11-15]. Here, we have investigated the efficacy of this treatment combination using a meta-analysis. Meta-analyses can reduce potential sample bias and increase statistical power in comparison to single studies with small samples.

\section{Aim}

This meta-analysis aimed to analyse the clinical efficacy of stenting with HIFU ablation for patients with distal MBO caused by PC.

\section{Material and methods}

This meta-analysis was conducted in accordance with the Preferred Reporting Items for Systematic reviews and Meta-Analyses (PRISMA) reporting guidelines [16]. Databases, including PubMed, Cochrane Library, Embase, Wanfang, VIP, and CNKI, were searched for relevant articles published through March 2021 based on the following search strategy: (((biliary [Title/Abstract]) AND (((stent [Title/Abstract]) OR (SEMS [Title/Abstract])) OR (drainage [Title/Abstract]))) AND (((obstruction [Title/Abstract]) OR (stenosis [Title/Abstract])) OR (stricture [Title/Abstract]))) AND ((high-intensity focused ultrasound [Title/Abstract]) OR (HIFU [Title/Abstract])). This work was registered in https://inplasy.com/ (No. INPLASY 202130047).

Study inclusion criteria were as follows: type of study: comparative studies; disease: distal MBO caused by PC; types of intervention: biliary stenting versus biliary stenting with HIFU ablation; language: any.

The following types of studies were excluded: (a) single-arm studies; (b) case reports; (c) animal studies; (d) review articles.

\section{Data}

Data including publication year, study design, authors, baseline patient characteristics, and treatment information were extracted from the identified studies by 2 independent investigators. Any disputes were resolved by consultation with a third investigator.

\section{Quality assessment}

The potential bias in randomized controlled trials (RCTs) was assessed by the Cochrane risk of bias tool for bias risks in detection, selection, reporting, attrition, and performance, amongst others.

Non-RCT studies were assessed with the 9-point Newcastle-Ottawa scale [17]. Scores of < 4, 4-6, and $\geq 7$ represented high, moderate, and low bias risks, respectively.

\section{Endpoints and definitions}

Stent dysfunction was the primary endpoint. Secondary endpoints included improvement of total bilirubin (TBIL), stent patency, clinical response rate to HIFU, OS, and complications.

Stent dysfunction was defined as the migration or re-obstruction of the stent. A positive clinical response to HIFU was the detection of tumour necrosis or reduction on computed tomography (CT) assessment [11-15]. OS was defined as the interval between stent insertion and death.

\section{Statistical analysis}

RevMan v5.3 and Stata v12.0 were utilized for all statistical analyses. The pooled odds ratios (ORs) and 95\% confidence intervals (Cls) for dichotomous variables were calculated using the Mantel-Haenszel approach, while pooled estimates of the mean difference (MD) with $95 \% \mathrm{Cls}$ were determined for continuous variables. Stent patency and OS between groups were compared using hazard ratios (HRs) with 95\% Cls. Heterogeneity among the included studies was gauged using the $\chi^{2}$ test and the $I^{2}$ statistic $\left(I^{2}>50 \%\right.$ indicating significant heterogeneity). Fixed-effects models were used in the absence of any significant heterogeneity. Sources of potential heterogeneity were identified via sensitivity analysis. The pooled clinical response rates were calculated using Stata v12.0. Funnel plots were used to analyse the potential publication bias.

\section{Results}

\section{Studies}

Twenty-nine potentially relevant reports were initially identified, and 5 of these were finally included (Figure 1). Four studies were retrospective [11-14], and one was an RCT [15]. These studies included 142 patients who underwent biliary stenting alone and 132 patients who underwent biliary stenting with HIFU ablation (Table I). All the stents were self-expanding metal stents with a diameter of $8 \mathrm{~mm}$.

The included RCT was an open-label study. The outcome assessment blinding, selective reporting, and other potential biases were unclear for this included RCT [15] (Figure 2). Newcastle-Ottawa scale scores between 7 and 8 were found for the retrospective studies (Table II).

\section{Improvements in TBIL}

Three studies reported data on TBIL improvement [13-15]. The pooled $\Delta$ TBIL values were similar between the 2 groups ( $p=0.10$, Figure $3 A$ ). These studies showed significant heterogeneity $\left(R^{2}=66 \%\right)$, which disappeared $\left(R^{2}=0 \%\right)$ after exclusion of Yang et al. [15]. 


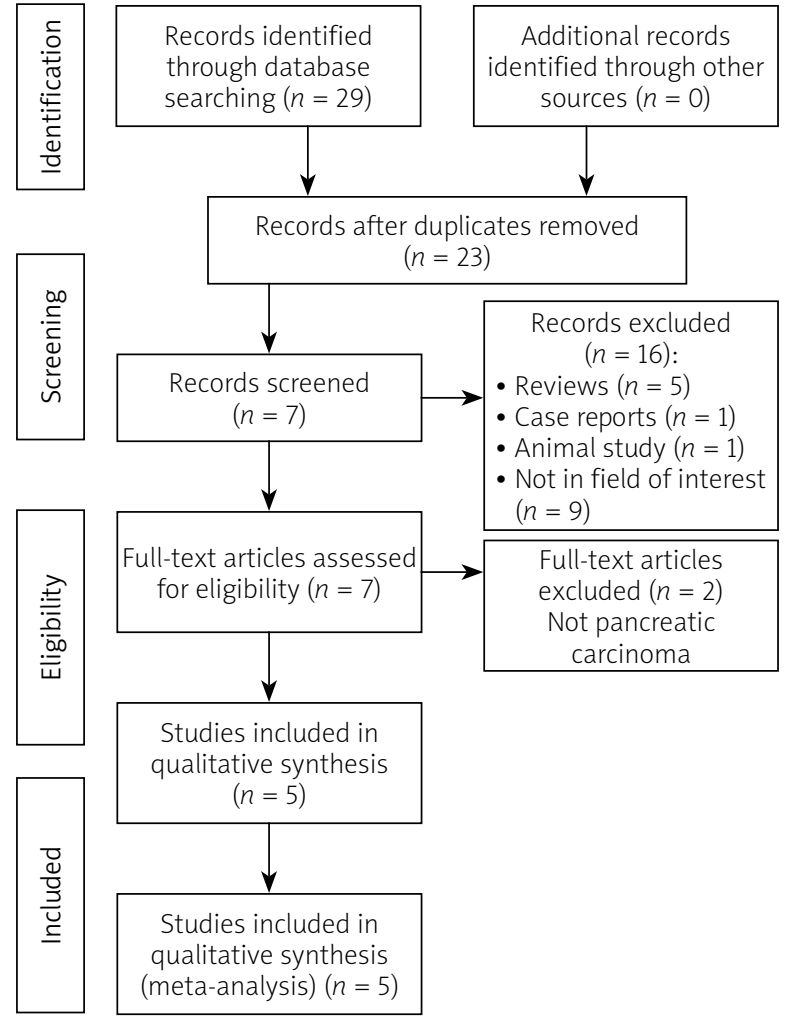

Figure 1. Flowchart of this meta-analysis

The pooled $\triangle T B I L$ values between the groups remained comparable after the removal of the study $(p=0.26)$.

\section{Stent dysfunction}

Data on stent dysfunction were included in all studies. The pooled rate of stent dysfunction was significantly greater in the stenting alone group $(30.3 \%$ vs. $18.9 \%, p=0.03$, Figure $3 \mathrm{~B})$. No significant heterogeneity was seen among these studies $\left(R^{2}=0 \%\right)$.

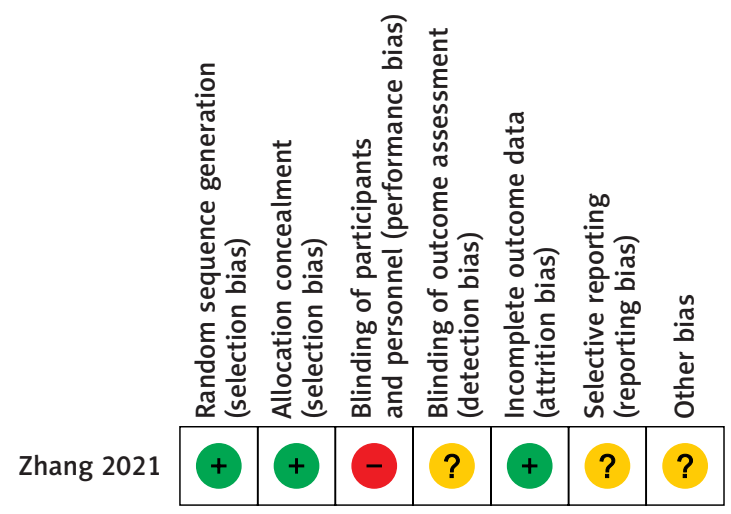

Figure 2. The Cochrane risk of bias tool for the included RCT

\section{Stent patency}

Two studies reported data on the duration of stent patency $[14,15]$. The pooled HR for stent patency duration indicated that the patency duration was longer in the stenting with HIFU ablation group ( $\mathrm{HR}=2.18$; $95 \% \mathrm{Cl}: 1.65-2.88, p<0.0001$, Figure $3 \mathrm{C}$ ). No significant heterogeneity was observed among these studies $\left(R^{2}=0 \%\right)$.

\section{OS}

Three studies reported OS data [13-15]. The pooled HR for OS indicates that the OS was longer in the stenting with $\mathrm{HIFU}$ ablation group $(\mathrm{HR}=2.37 ; 95 \% \mathrm{Cl}$ : $1.87-3.01, p<0.0001$, Figure $3 \mathrm{D})$. No significant heterogeneity was found among these reports $\left(R^{2}=0 \%\right)$.

\section{Clinical response to HIFU}

All studies reported data on clinical response to HIFU. We found HIFU ablation to be associated with an

Table I. Characteristics of the included studies

\begin{tabular}{|c|c|c|c|c|c|c|c|c|}
\hline Study/year/country & Design & NOS & $\begin{array}{l}\text { Stent } \\
\text { type }\end{array}$ & Groups & Sample size & Age [years] & $\begin{array}{l}\text { Cancer } \\
\text { stage }\end{array}$ & ECOG PS \\
\hline \multirow[t]{2}{*}{ Cao/2010/China [11] } & \multirow[t]{2}{*}{ Retrospective } & \multirow[t]{2}{*}{7} & \multirow[t]{2}{*}{ Metal } & Stent & 6 & Not given & Not given & Not given \\
\hline & & & & Stent + HIFU & 5 & Not given & Not given & Not given \\
\hline \multirow[t]{2}{*}{ Niu/2016/China [12] } & \multirow[t]{2}{*}{ Retrospective } & \multirow[t]{2}{*}{7} & \multirow[t]{2}{*}{ Metal } & Stent & 7 & Not given & Not given & $0,1, \geq 2$ \\
\hline & & & & Stent + HIFU & 9 & Not given & Not given & $0,1, \geq 2$ \\
\hline \multirow[t]{2}{*}{ Xia/2017/China [13] } & \multirow[t]{2}{*}{ Retrospective } & \multirow[t]{2}{*}{8} & \multirow[t]{2}{*}{ Metal } & Stent & 42 & 63.6 & II-IV & $2-3$ \\
\hline & & & & Stent + HIFU & 38 & 64.6 & II-IV & $2-3$ \\
\hline \multirow[t]{2}{*}{ Yang/2019/China [14] } & \multirow[t]{2}{*}{ Retrospective } & \multirow[t]{2}{*}{8} & \multirow[t]{2}{*}{ Metal } & Stent & 41 & 63.6 & II-IV & $2-3$ \\
\hline & & & & Stent + HIFU & 34 & 65.2 & II-IV & $2-3$ \\
\hline \multirow[t]{2}{*}{ Zhang/2021/China [15] } & \multirow{2}{*}{$\begin{array}{l}\text { Randomized } \\
\text { controlled trial }\end{array}$} & \multirow[t]{2}{*}{-} & \multirow[t]{2}{*}{ Metal } & Stent & 46 & 63.2 & II-IV & 2.5 \\
\hline & & & & Stent + HIFU & 46 & 64.9 & II-IV & 2.5 \\
\hline
\end{tabular}

NOS - Newcastle-Ottawa score, HIFU - high-intensity-focused ultrasound, ECOG PS - Eastern Cooperative Oncology Group performance status. 
Table II. Characteristics of the treatment outcomes

\begin{tabular}{lccccccc} 
Study & Groups & $\Delta$ TBIL $[\boldsymbol{\mu m o l} / \mathbf{l}]$ & $\begin{array}{c}\text { Stent } \\
\text { dysfunction }\end{array}$ & Patency & Cholangitis & Pancreatitis & Survival \\
\hline Cao [11] & Stent & Not given & $3 / 6(50 \%)$ & Not given & Not given & Not given & Not given \\
\cline { 2 - 8 } & Stent + HIFU & Not given & $1 / 5(20 \%)$ & Not given & Not given & Not given & Not given \\
\hline \multirow{2}{*}{ Niu [12] } & Stent & Not given & $3 / 7(42.9 \%)$ & Not given & Not given & Not given & Not given \\
\cline { 2 - 8 } & Stent + HIFU & Not given & $2 / 9(22.2 \%)$ & Not given & Not given & Not given & Not given \\
\hline \multirow{2}{*}{ Xia [13] } & Stent & 106.6 & $9 / 42(21.4 \%)$ & Not given & Not given & Not given & $145 \mathrm{~d}$ \\
\cline { 2 - 8 } & Stent + HIFU & 105.6 & $6 / 38(15.8 \%)$ & Not given & Not given & Not given & $209 \mathrm{~d}$ \\
\hline Yang [14] & Stent & 103.1 & $13 / 41(31.7 \%)$ & 118 & $5 / 41(12.2 \%)$ & 0/41 (0\%) & $118 \mathrm{~d}$ \\
\cline { 2 - 8 } & Stent + HIFU & 146.4 & $7 / 34(20.6 \%)$ & 175 & $3 / 34(8.8 \%)$ & $1 / 34(2.9 \%)$ & $175 \mathrm{~d}$ \\
\hline Zhang [15] & Stent & 103.8 & $15 / 46(32.6 \%)$ & 120 & $9 / 46(19.6 \%)$ & $1 / 46(2.2 \%)$ & $140 \mathrm{~d}$ \\
\cline { 2 - 8 } & Stent + HIFU & 119.7 & $9 / 46(19.6 \%)$ & 188 & $7 / 46(15.2 \%)$ & $1 / 46(2.2 \%)$ & $218 \mathrm{~d}$
\end{tabular}

HIFU - high-intensity-focused ultrasound, TBIL - total bilirubin.

A

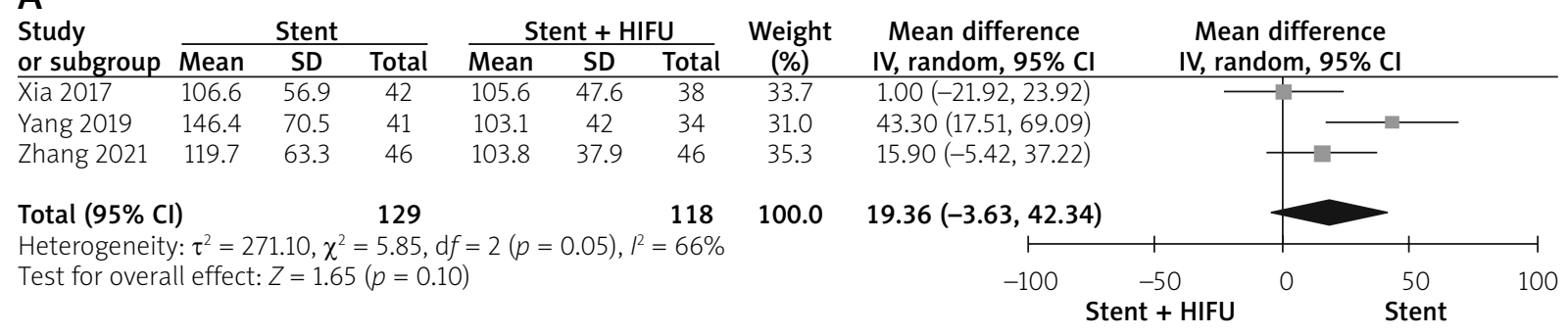

B

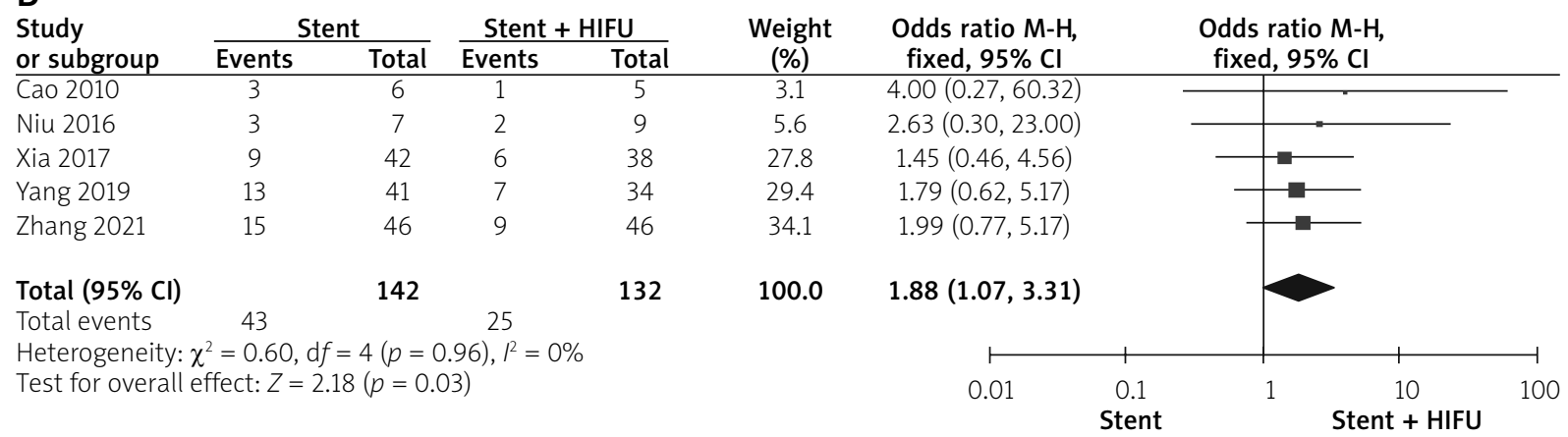

\section{C}

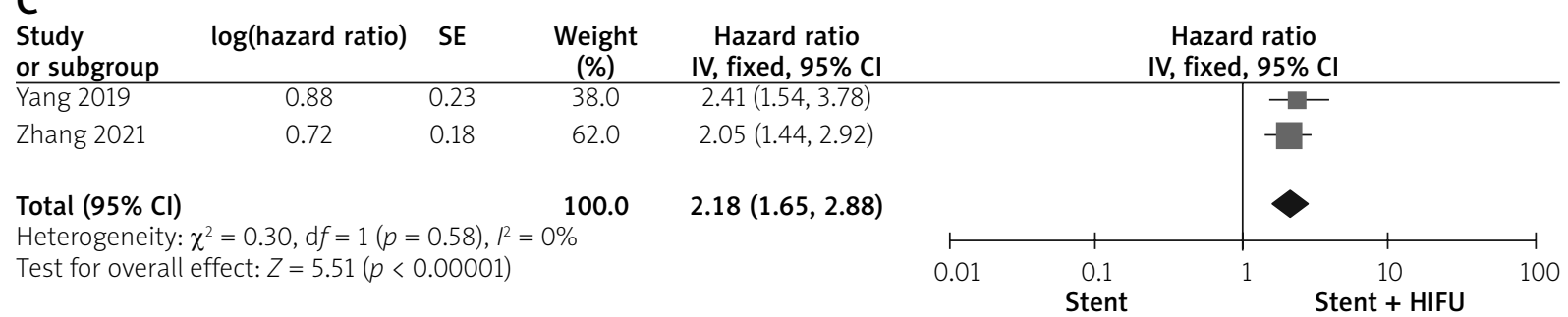

Figure 3. Forest plots of improvement of TBIL (A), stent dysfunction rates (B), stent patency duration (C) 
D

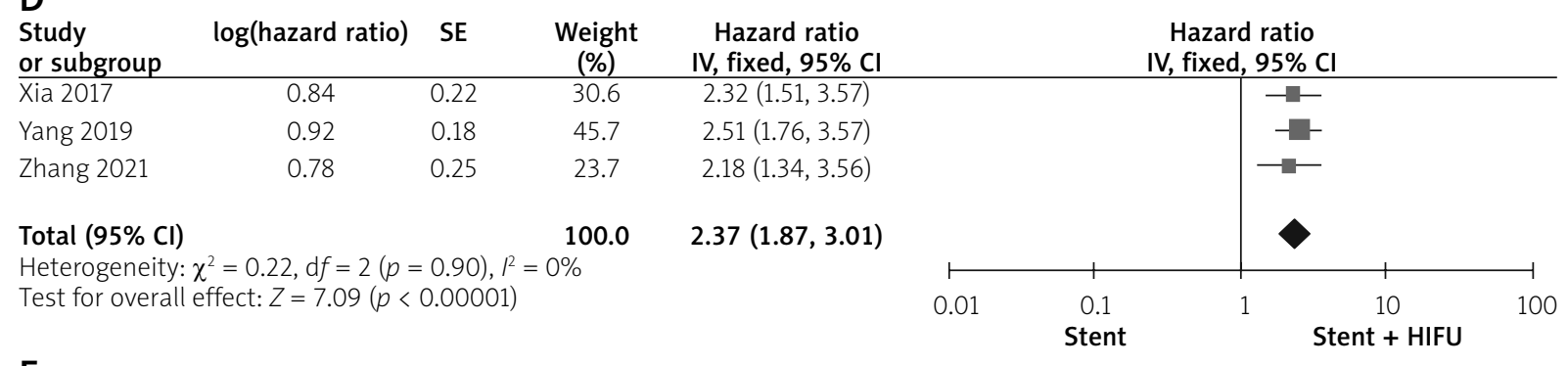

\section{$E$}

Study ID

ES $(95 \% \mathrm{Cl})$

Weight (\%)

Cao 2010

Niu 2016

Xia 2017

Yang 2019

Zhang 2021

Overall $\left(I^{2}=0.0 \%, p=0.893\right)$

Note: Weight are from random effects analysis.

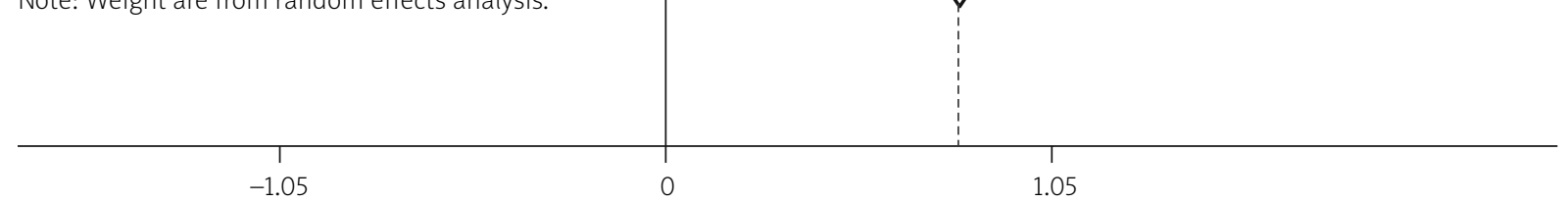

$\mathrm{F}$

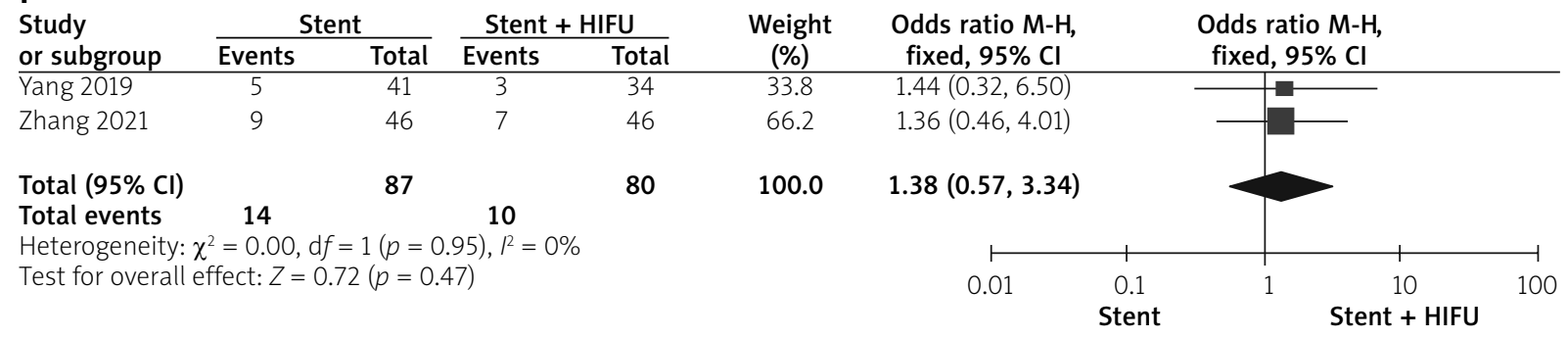

G

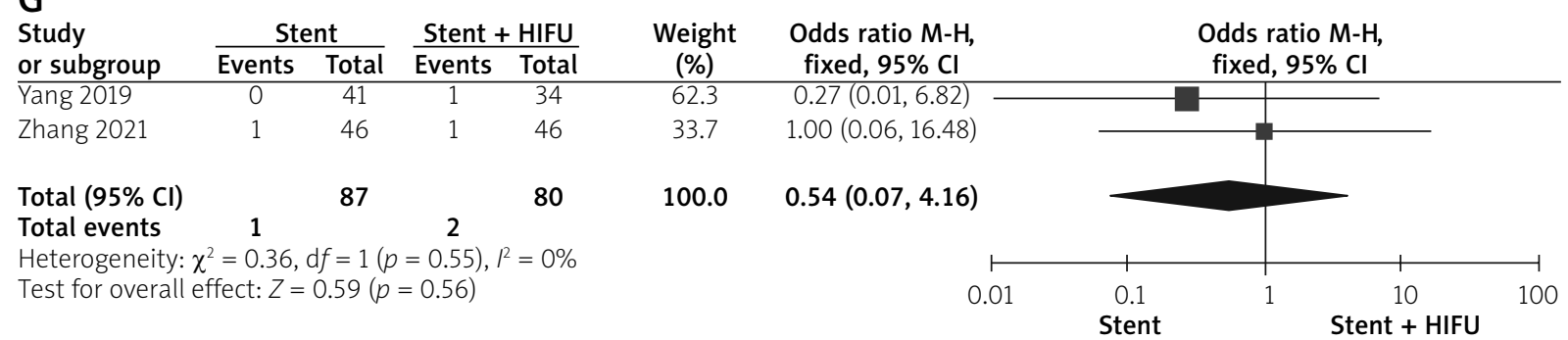

Figure 3. Cont. OS (D), clinical response rates (E), cholangitis rates (F), and pancreatitis rates (G) 
$80 \%$ pooled clinical response rate (Figure $3 \mathrm{E}$ ). These reports showed no significant heterogeneity $\left(I^{2}=0 \%\right)$.

\section{Complications}

Two studies reported data on the rates of cholangitis and pancreatitis $[14,15]$. The pooled cholangitis rates were comparable between the 2 groups $(16.1 \%$ vs. $12.5 \%, p=0.47$, Figure $3 \mathrm{~F}$ ), with no significant heterogeneity observed between the studies $\left(I^{2}=0 \%\right)$. The pooled pancreatitis rates were comparable between the 2 groups ( $1.1 \%$ vs. $2.5 \%, p=0.56$, Figure $3 \mathrm{G})$, again with no significant between-study heterogeneity $\left(R^{2}=0 \%\right)$.

\section{Publication bias}

Funnel plots did not detect any evidence of publication bias connected to the study endpoints.

\section{Discussion}

Here, we assessed the clinical efficacy of biliary stenting with HIFU ablation in patients with distal MBO caused by PC. The results indicated that the patients' stent dysfunction, stent patency duration, and OS were significantly improved by the HIFU ablation.

Initially, we observed similar pooled $\triangle T B I L$ values between the 2 groups, indicating that HIFU ablation does not influence the short-term effectiveness of biliary stenting.

Biliary stents have previously been reported to be associated with $50 \%$ cumulative 6 -month re-obstruction rates [18]. While some studies have found that covered stents have longer patency in distal MBO patients than uncovered stents $[6,9]$, these covered stents do not slow the cancer progression. Covered stent insertion has also been related to higher rates of cholecystitis and pancreatitis in treated patients $[6,7]$.

Here, the stent patency function was assessed by calculating the rate of stent dysfunction and the duration of stent patency. We found that both the pooled stent dysfunction rate $(p=0.03)$ and the stent patency duration $(p<0.0001)$ were favourable in the stenting with HIFU group. These results indicate that HIFU ablation can effectively halt tumour progression and prolong stent patency. However, in these included studies, although the stent dysfunction rates were lower in the stenting with HIFU group, this difference was not significant [11-15]. When these rates were pooled by the meta-analysis, the statistical power was increased and the difference became significant.

All the included studies used $8 \mathrm{~mm}$ diameter metal stents [11-15]. Metal stents appear to be superior to plastic in achieving long-term patency duration [3]. Several studies have used $10 \mathrm{~mm}$ stents for MBO patients $[19,20]$; however, a recent report showed comparable stent patency, survival duration, and complication rates for $8 \mathrm{~mm}$ and $10 \mathrm{~mm}$ stents in MBO [21].

We found significantly longer pooled OS rates for patients receiving stenting with HIFU ablation compared to stenting alone. This is consistent with prior studies that have found that anticancer treatment can prolong survival in patients who have undergone stent insertion [8, 9, 22]. HIFU ablation can improve stent patency and OS in patients with distal MBO caused by PC because PC is a mass-like tumour. In contrast to distal MBO caused by the lumen-like cholangiocarcinoma, PC can be treated using specialized equipment to focus the ultrasound energy on a specific target within the body, thereby driving thermally induced apoptosis and necrosis [23]. We also determined that HIFU ablation was associated with an $80 \%$ pooled clinical response rate. This finding, together with the OS-related results, suggests that HIFU ablation may be well-suited to the treatment of distal MBO caused by PC.

No differences in cholangitis and pancreatitis rates were detected between the 2 patient groups, suggesting that HIFU ablation is not associated with an increased complication rate. However, only 2 of the included studies included data on cholangitis and pancreatitis rates, indicating the necessity of further studies to confirm these results.

The study has several limitations. Firstly, the retrospective nature of many of the included studies may introduce bias. Second, although this meta-analysis only included the studies that focused on distal MBO caused by PC, several studies did not provide the details of the tumour stage and the Eastern Cooperative Oncology Group performance status, which may increase bias risk. Third, the included studies were all conducted in China. These results, therefore, need to be verified in other populations.

\section{Conclusions}

This meta-analysis demonstrated that stenting accompanied by HIFU ablation increased both stent patency and OS in patients with distal MBO caused by PC compared to stenting alone.

\section{Acknowledgments}

Program of 2019 Xuzhou clinical technique research (2109GG002).

\section{Conflict of interest}

The authors declare no conflict of interest.

\section{References}

1. Khoo S, Do NDT, Kongkam P. Efficacy and safety of EUS biliary drainage in malignant distal and hilar biliary obstruction: 
a comprehensive review of literature and algorithm. Endosc Ultrasound 2020; 9: 369-79.

2. Matsumoto K, Takeda Y, Onoyama T, et al. Endoscopic treatment for distal malignant biliary obstruction. Ann Transl Med 2017; $5: 190$.

3. Fernandez Y, Viesca M, Arvanitakis M. Early diagnosis and management of malignant distal biliary obstruction: a review on current recommendations and guidelines. Clin Exp Gastroenterol 2019; 12: 415-32.

4. Zu QQ, Zhang JX, Wang B, et al. Percutaneous transpapillary biliary stent placement for distal malignant biliary obstruction: outcomes and survival analysis. Turk J Gastroenterol 2019; 30 714-21.

5. Miller CS, Barkun AN, Martel M, et al. Endoscopic ultrasound-guided biliary drainage for distal malignant obstruction: a systematic review and meta-analysis of randomized trials. Endosc Int Open 2019; 7: E1563-73.

6. Yang MJ, Kim JH, Yoo BM, et al. Partially covered versus uncovered self-expandable nitinol stents with anti-migration properties for the palliation of malignant distal biliary obstruction a randomized controlled trial. Scand J Gastroenterol 2015; 50: 1490-9.

7. Sampaziotis F, Elias J, Gelson WT, et al. A retrospective study assessing fully covered metal stents as first-line management for malignant biliary strictures. Eur J Gastroenterol Hepatol 2015; 27: 1347-53.

8. Zhu HD, Guo JH, Zhu GY, et al. A novel biliary stent loaded with (125)I seeds in patients with malignant biliary obstruction: preliminary results versus a conventional biliary stent. J Hepatol 2012; 56: 1104-11.

9. Kitano M, Yamashita Y, Tanaka K, et al. Covered self-expandable metal stents with an anti-migration system improve patency duration without increased complications compared with uncovered stents for distal biliary obstruction caused by pancreatic carcinoma: a randomized multicenter trial. Am J Gastroenterol 2013; 108: 1713-22.

10. Yokota Y, Fukasawa M, Takano S, et al. Partially covered metal stents have longer patency than uncovered and fully covered metal stents in the management of distal malignant biliary obstruction: a retrospective study. BMC Gastroenterol 2017; 17: 105

11. Cao C, Cheng L, Li GJ, et al. Biliary stent implantation combined with high intensity-focused ultrasound ablation for the treatment of malignant biliary obstruction. J Interv Radiol 2011; 20: 792-5.

12. Niu S, Cheng L, Qiao Y, et al. Combined stent insertion and high-intensity focused ultrasound ablation for patients with malignant obstructive jaundice. Surg Laparosc Endosc Percu$\tan$ Tech 2016; 26: 488-92.

13. Xia H, Zhou C, Gong Z. Stent insertion combined with high-intensity focused ultrasound ablation for malignant obstructive jaundice caused by pancreatic carcinoma. J Reg Anat Oper Surg 2017; 26: 698-701.

14. Yang SY, Liu F, Liu Y, et al. Stent insertion with high-intensity focused ultrasound ablation for distal biliary obstruction secondary to pancreatic carcinoma. Medicine 2020; 99: e19099.

15. Zhang FQ, Li L, Huang PC, et al. Stent insertion with high intensity-focused ultrasound ablation for biliary obstruction caused by pancreatic carcinoma: a randomized controlled trial. Surg Laparosc Endosc Percutan Tech 2021; 31: 298-303.

16. Liberati A, Altman DG, Tetzlaff J, et al. The PRISMA statement for reporting systematic reviews and meta-analyses of studies that evaluate health care interventions: explanation and elaboration. PLoS Med 2009; 6: e1000100.

17. Benatar JR, Stewart RAH. Cardiometabolic risk factors in vegans: a meta-analysis of observational studies. PLoS One 2018; 13: e0209086.

18. Loew BJ, Howell DA, Sanders MK, et al. Comparative performance of uncoated, self-expanding metal biliary stents of different designs in 2 diameters: final results of an international multicenter, randomized, controlled trial. Gastrointest Endosc 2009; 70: 445-53.

19. Gwon DI, Ko GY, Sung KB, et al. Percutaneous biliary metallic stent placement in patients with unilobar portal vein occlusion caused by advanced hilar malignancy: outcome of unilateral versus bilateral stenting. AJR Am J Roentgenol 2011; 197: 795-801.

20. Naitoh I, Ohara H, Nakazawa T, et al. Unilateral versus bilateral endoscopic metal stenting for malignant hilar biliary obstruction. J Gastroenterol Hepatol 2009; 24: 552-7.

21. Kawashima H, Hashimoto S, Ohno E, et al. Comparison of 8- and 10-mm diameter fully covered self-expandable metal stents: a multicenter prospective study in patients with distal malignant biliary obstruction. Dig Endosc 2019; 31: 439-47.

22. Sha KH, Liu TG, Yang F, et al. Irradiation stent insertion for inoperable malignant biliary obstruction: a meta-analysis of randomized controlled trials. Abdom Radiol 2021; 46: 2173-81.

23. Wu F. High intensity focused ultrasound: a noninvasive therapy for locally advanced pancreatic cancer. World J Gastroenterol 2014; 20: 16480-8.

Received: 21.05.2021

Accepted: 6.06 .2021 